\title{
A Principal Component Analysis Control Chart Method for Catenary Status Evaluation and Diagnosis
}

\author{
Shan Lin $\left(\mathbb{D},{ }^{1}\right.$ Liping Liu $\mathbb{D}^{1},{ }^{1}$ Meiwan Rao $\mathbb{D}^{1},{ }^{1}$ Shu Deng $\mathbb{D},{ }^{1}$ Jiaxin Wang $\left(\mathbb{D},{ }^{2}\right.$ \\ Wenfan Zhong $\mathbb{D}{ }^{2}$ and Li Lun $\mathbb{D}^{2}$ \\ ${ }^{1}$ Guangzhou Metro Design«Research Institute Co., Ltd., Guangzhou 510010, China \\ ${ }^{2}$ School of Electrical and Automation, EastChina Jiaotong University, Nanchang 330013, China \\ Correspondence should be addressed to Li Lun; 664313727@qq.com
}

Received 20 October 2021; Accepted 13 December 2021; Published 24 December 2021

Academic Editor: Yuan Mei

Copyright () 2021 Shan Lin et al. This is an open access article distributed under the Creative Commons Attribution License, which permits unrestricted use, distribution, and reproduction in any medium, provided the original work is properly cited.

To make accurate and comprehensive evaluation of the catenary and diagnose the causes of the catenary fault, a method of catenary state evaluation and diagnosis based on the principal component analysis control chart was proposed, which can make full use of the multidimensional detection parameters of the catenary. The principal component analysis was used to reduce the dimension of catenary parameters, the principal component $T^{2}$ control chart was calculated to show the change of principal component of catenary state data, the residual SPE control chart was calculated to show the change of their correlation, and the contribution rate control chart was calculated to show the cause of abnormal state data. The method can not only transform the multidimensional detection parameters of the catenary into a statistic to realize the simple and intuitive evaluation of the catenary state but also can accurately determine the cause of the abnormal state, so as to provide technical support for the targeted condition-based maintenance of the catenary.

\section{Introduction}

As the only power supply line for electrified railway, catenary's working environment is harsh, and there is no backup for it, once there is a fault, it will lead to the outage of electrified railway, which will have a huge impact on the railway operation. As a special power supply line, catenary has the following characteristics:

(1) Operating environment is unique. Catenary is erected along the railway, exposed to the air, and is subject to the high-speed impact of locomotive pantograph, the space environment, climate environment, and working environment are unique compared to ordinary transmission lines, which makes the catenary more prone to failure and is greatly affected by the external environment.

(2) There is no backup for catenary. Due to the particularity of the catenary operating environment, there is no backup for catenary, Once the catenary is abnormal, it will lead the electrified railway to fail, resulting in huge economic losses.

(3) There is electromechanical compound effect for catenary. As a complex mechanical structure, the catenary's main function is to ensure a good and stable power supply, it needs to maintain structural stability under various mechanical loads and electrical shocks, thus to provide a good and stable current to the electric locomotive.

(4) There are moving loads for catenary. The pantograph of electric locomotive gets energy by sliding through the catenary, the load of the catenary fluctuates with time, and its position moves dynamically.

The statistical data show that the failure of the traction power supply system is mainly caused by the catenary failure, which accounts for more than $90 \%$ of the failure of the traction power supply system [1]. In order to avoid safety and economic losses caused by catenary failures, it needs to maintain the catenary in good working condition. Accurate 
evaluation and diagnosis of the catenary status are the prerequisites for realizing the maintenance of the catenary and keeping the catenary in good condition.

The state detection of catenary includes static detection and dynamic detection. Static detection is a routine detection of manual use of portable detection equipment. Dynamic detection measures the parameters of the catenary under the actual operation state by the detection equipment installed on the special detection vehicle.

Static detection is carried out manually, which is intensive, time-consuming, inefficient, and is limited by the time of the skylight. A multifunctional laser measuring instrument was proposed in [2] for the static detection. The multivision technology was proposed to determine the catenary according to the image characteristics captured by the camera and to analyse the pantograph catenary components through intelligent image recognition [3].

Since the catenary is a flexible mechanical suspension system, the pantograph will cause the catenary to rise, so the dynamic parameters of the catenary are different from the static parameters. In the 1950s, Germany and Japan began to develop catenary detection vehicles, which installed various sensors and other equipment on the roof of locomotives, so as to detect the pull-out value and height under dynamic conditions when the train was running [4-6]. With the rapid development of high-speed railways, the relationship between the pantograph and the catenary has gradually become complicated, the catenary detection parameters have also expanded from the height and pull-out value to the pantograph contact force, the vertical acceleration of the pantograph head, and the off-line rate.

China proposes to build a traction power supply safety detection and monitoring system (6C system), which aim to achieve comprehensive detection and monitoring of the traction power supply system in all directions and full coverage [7-10]. The catenary information obtained by the 6C system is more diversified, in addition to the traditional geometric detection parameters of the catenary, it also includes various high-definition pictures, videos, and infrared detection information, and this unstructured information can be processed to extract the structural information of the catenary.

It can be seen that with the development of detection technology, the detection parameters of catenary become more and more comprehensive. How to effectively use these detection data to comprehensively and accurately evaluate the state of the catenary has become a new problem.

At present, the single-threshold comparison method is used to evaluate the state of catenary, which compares each parameter with the corresponding standard value to determine whether a certain parameter of the catenary exceeds the standard [11]. Commonly used catenary parameters include pull-out value and height [12], as well as dynamic parameters such as height difference, hard point, and contact force $[1,13]$. With the increasing number of catenary detection parameters, this single-parameter comparison method is inefficient in judgment, and the judgment method is simple, which cannot meet the needs of comprehensive evaluation of the multidimensional parameters of the catenary. At the same time, the single-parameter comparison method does not consider the correlation between the catenary parameters, and there may be conflicting judgment results in some conditions [14].

Different detection parameters reflect the state of the same catenary from different respects. The comprehensive evaluation of the multidimensional parameters can obtain a more comprehensive and accurate evaluation result of the catenary status. For example, the operation quality index CQI was proposed to evaluate the operation quality of the catenary [15], but the index does not consider the influence degree difference of different parameters. On the basis of CQI, [16] used the analytic hierarchy process to determine the weight of each indicator, but it need to specify the importance of each indicator manually, which was highly subjective. In order to use the objective law embodied by the catenary parameters, the entropy method was proposed to determine the weight through the law embodied by the change of the catenary parameters $[17,18]$, and the fuzzy comprehensive evaluation method was proposed to evaluate the state of the catenary comprehensively. A combination method that combines subjective and objective methods to perform hybrid calculations on the weights of indicators in [19-21]. The above method can determine the degree of influence of catenary parameters on the state of the catenary from a subjective or objective perspective, but the calculation is complicated, and the results are not intuitive enough to reflect the inherent relationship between the catenary parameters. For this reason, [22] carried out cluster analysis on the catenary detection parameters and performed linear regression on each type of data, so as to obtain the mathematical model of the catenary detection parameters, and judge the state of the catenary based on the regression model. The normal cloud model was proposed in $[23,24]$ to process the detection index, solve the problem of ambiguity and randomness of the evaluation index, and establish a comprehensive evaluation model of the catenary operating state. The set pair analysis method was proposed to determine the degree of connection between each evaluation index and the health status level [25]. These methods can carry out a graded evaluation of the catenary status, but the evaluation results cannot reflect the cause of the abnormal status, so they cannot provide targeted guidance for the catenary maintenance.

Normally, the detection parameters of the catenary fluctuate around its standard value, and there is a certain correlation between the parameters. When the state of the catenary is abnormal, the state parameters of the catenary will deviate from the standard value. At the same time, due to the abnormality of the catenary structure, the original correlation of the catenary parameters will be destroyed. Therefore, the degree of deviation of the catenary detection parameters from the standard value and the change in the correlation between the catenary detection parameters can reflect the abnormality of the catenary status.

Multivariate statistical analysis is a method of comprehensive analysis of multidimensional data, which can analyse the statistical distribution rules and interrelationships of multidimensional parameters. The multivariate statistical 
control chart based on multivariate statistical analysis is a commonly used quality management tool [26]. It can directly reflect the change process of detection parameters in graphical form and comprehensively monitor, control, analyse, and evaluate the process with multivariate parameters [27]. This paper combines principal component analysis with multivariate statistical control charts and uses principal component analysis to reduce the dimensions of the multidimensional state parameters of the catenary. By obtaining the principal component space and residual space of the catenary detection parameters, the principal component $T^{2}$ control chart and the residual SPE control chart of the catenary detection parameters on this basis are established. The main element $T^{2}$ control chart and the residual SPE control chart are used to comprehensively evaluate the status of the catenary and analyse the reasons for the abnormal status of the catenary. The results obtained can be used for targeted guidance on the maintenance of the catenary.

\section{Multivariate Statistical Analysis of Catenary Status Based on Principal Component}

2.1. Principal Component Analysis. The detection parameters of the catenary are numerous and related to each other. In order to reduce the complexity of processing the catenary detection data and reduce the computational workload, principal component analysis is used to simplify and compares the catenary detection data. The original data space is transformed into the main element subspace and the error subspace to achieve the purpose of data dimensionality reduction. Among them, the principal component subspace is the principal component, which contains most of the data information; the error subspace is the space orthogonal to the principal component subspace and represents the degree of deviation of the data from the principal component subspace [28].

The steps to transform the catenary detection data into the main element subspace and the error subspace are as follows:

(1) Standardize the test data. Due to the differences between the dimensions of each parameter lead to too large deviations between the data, it is necessary to standardize the detection parameters first to obtain the standardized detection data $X$;

(2) Calculate the correlation coefficient matrix:

$$
\sum=X \cdot X^{T} .
$$

(3) Calculate the principal components and load matrix. Find the characteristic equation:

$$
\left|\sum-\lambda I\right|=0
$$

Among them, $I$ is the identity matrix. Solve the $p$ eigenvalues and their corresponding eigenvectors and arrange the eigenvalues in descending order: $\lambda_{1} \geq \lambda_{2} \geq, \ldots, \geq \lambda_{p}$, where $p$ is the dimension of the detection parameter.
(4) Determine the number of principal components of the principal component subspace according to the cumulative contribution rate. When the cumulative sum of eigenvalues is greater than a certain specified value $C_{R}$, the selected data information of the $k$ principal components can already include most of the data information, that is, satisfy:

$$
\sum_{l=1}^{k}\left(\frac{\lambda_{l}}{\sum_{i=1}^{p} \lambda_{i}}\right) \geq C_{R} .
$$

(5) Determine the load matrix $P$ and the principal component space $t$. The selected eigenvectors corresponding to the $k$ principal components form the load matrix $P$, and the principal component space $t$ of the detection data is expressed as

$$
t=X P \text {. }
$$

(6) Calculate the score matrix. The score matrix $\widehat{X}$ is the projection of the standardized data $X$ in the principal component space $t$ :

$$
\stackrel{\wedge}{X}=t P^{T} .
$$

Since there is an error between the projection of the data in the principal component space and the actual data, that is, there is an error subspace $E$ in the actual model space and the principal component subspace, the original data can be expressed as

$$
X=\stackrel{X}{X}+E .
$$

According to (5) and (6), the error space $E$ under the action of the principal component space:

$$
\begin{aligned}
E & =X-\widehat{X} \\
& =X-t P^{T} \\
& =X-X P P^{T} .
\end{aligned}
$$

It can be seen from (7) that the principal component space reduces the $p$-dimension detection parameters of the original data to $k$-dimension, and there is no correlation between the data. The remaining $(p-k)$ dimensional data constitute the error subspace $E$, which has not undergone principal component transformation, and $E$ contains the correlation information of the detection parameters.

\subsection{Evaluation of Catenary Status Based on Principal Com-} ponents Control Chart. The control chart is a quality control tool that can monitor and diagnose the process. In order to display the catenary status more intuitively and reflect the development trend of the catenary status, the principal component analysis and the multivariate statistical control chart are combined to obtain the multivariate statistical control chart based on the principal component analysis, which includes principal component $T^{2}$ control chart, 
residual SPE control chart, and principal component contribution control chart [29].

The main component $T^{2}$ control chart uses the $T^{2}$ statistics to reflect the change of the main metadata, which can realize the status judgment of the main metadata of the detected data. The SPE control chart uses the residual SPE statistics for statistical testing, which reflects the degree of deviation of the data from the pivot space and can reflect the changes in the correlation between the data. The principal component contribution control chart calculates the contribution rate of each parameter at the abnormal point to the $T^{2}$ statistic and the SPE statistic, which can reflect the cause of the abnormality and play a diagnostic role. Different from other types of control charts, the principal component analysis control chart can not only reflect the changes in state data but also accurately diagnose the cause of the abnormality when the state is abnormal, so as to provide guidance for maintenance.

2.2.1. Principal Component $T^{2}$ Control Chart. The principal component $T^{2}$ control chart is a statistical control chart that monitors the principal components in the principal component space based on the $T^{2}$ statistics [30]. It can reflect the change trend and the degree of deviation of the principal components after the dimension reduction of the catenary detection parameters and reflect the change of the data in the principal component space.

Based on the definition of Hotelling $T^{2}$ statistic, for the $i$ th detection group, its principal component $T_{i}^{2}$ statistic is expressed as

$$
T_{i}^{2}=t_{i} \Lambda^{-1} t_{i}^{\prime}
$$

Among them, $t_{i}$ represents the principal component of the $i$ th group, $\Lambda$ represents the diagonal matrix composed of the eigenvalues of the selected $k$ principal components. Since the principal component and the original data satisfy (4), it can be expressed in the form of the original data and load matrix:

$$
T_{i}^{2}=X_{i} P \Lambda^{-1} P^{T} X_{i}^{T} .
$$

According to (8) and (9), it can be seen that the principal component $T^{2}$ control chart is a manifestation of the change of principal component data in the principal component space on the basis of eliminating the correlation between the detection parameters of the catenary.

The control limit of the $T^{2}$ control chart is expressed as

$$
T^{2} U C L=\frac{k(m-1)}{m-k} F_{1-\alpha}(k, m-1) .
$$

Among them, $F_{1-\alpha}(k, m-1)$ represents the number of principal components of the first degree of freedom $k$, the second degree of freedom is $m-1, m$ is the number of detection parameter groups, and the confidence is the $F$ distribution of $\alpha$. When $T_{i}^{2}>T^{2} \mathrm{UCL}$, it means that the main component data fluctuate beyond the normal range, and the catenary status is abnormal.
2.3. Residual SPE Control Chart. The SPE control chart is a control chart that reflects the change of the error between the $k$ principal component information and the $p$ parameter information of the catenary detection parameters [31], which includes the change of the correlation between the catenary detection parameters.

Once the SPE control chart is abnormal in the evaluation process, it means that the deviation between the data and the principal component space is too large at this time, and the data are abnormal. Since the SPE control chart is an error value formed by integrating all parameters, the SPE control chart can not only monitor the deviation of the data relative to the principal element space but also detect the change in the internal correlation of the data. For the $i$ th sample, the SPE statistics are calculated as

$$
\begin{aligned}
Q_{i} & =E^{T} E \\
& =X_{i}^{T}\left(I-P P^{T}\right)^{T}\left(I-P P^{T}\right) X_{i} .
\end{aligned}
$$

It can be seen from (11) that the $Q_{i}$ statistic reflects the degree to which the data deviates from the pivot space and at the same time reflects the change in the correlation between the data. The control limit of the SPE control chart is [32]:

$$
\text { QUCL }=\theta_{1}\left[\frac{z_{1-\alpha} h_{0} \sqrt{2 \theta_{2}}}{\theta_{1}}+\frac{\theta_{2} h_{0}\left(h_{0}-1\right)}{\theta_{1}^{2}}+1\right]^{1 / h_{0}} .
$$

$$
\text { Among them, } \quad \theta_{i}=\sum_{j=n+1}^{p} \lambda_{j}^{i}(i=1,2,3) \text {, }
$$
$h_{0}=1-2 \theta_{1} \theta_{3} / 3 \theta_{2}^{2}, z_{1-\alpha}$ is the $1-\alpha$ quantile of the Gaussian distribution. When $Q_{i}>Q U C L$, it means that the nonprincipal component part has a large deviation, and the principal component model is out of control and needs to be adjusted.

When the status of the catenary is abnormal, the status parameters of the catenary will shift, and the correlation between the parameters will also change, which will cause the statistics of the $T^{2}$ control chart and SPE control chart to exceed the limit. Therefore, the main component $T^{2}$ control chart and the residual SPE control chart can be used to judge the status of the catenary.

\section{Catenary Status Diagnosis Based on Contribution Rate Control Chart}

The $T^{2}$ control chart and SPE control chart can reflect the abnormality of the catenary status, but cannot find out the cause of the abnormality. The contribution control chart calculates the sum of the contribution rate of each parameter at the abnormal point to the abnormality, so as to determine the cause of the abnormality [29].

The abnormality in the $T^{2}$ control chart is caused by the principal component, and the contribution value of the $j$ th detection parameter $X_{j}$ is

$$
\operatorname{CONTT}_{j}^{2}=\sum_{l=1}^{k} P_{j l} X_{j} \lambda_{l}^{-1}
$$

Among them, $\mathrm{CONTT}_{j}^{2}$ represents the contribution rate of the $j$ th detection parameter to the principal component 
$T^{2}, P_{j l}$ represents the value of the $l$ th principal component of the $j$ th detection parameter in the load matrix $P$, and $\lambda_{l}$ represents the eigenvalue corresponding to the $l$ th principal component.

For the SPE control chart, the contribution rate at the abnormal point is the error square value at the fault point, and the contribution rate of the $j$ th detection parameter to the SPE statistics is

$$
\text { CONTSPE }=\left(X_{j}^{T}\left(I-\mathrm{PP}^{T}\right)\right)^{2} .
$$

The higher the contribution rate of the parameter, the greater the impact on the abnormality of the fault, so the cause of the abnormality of the catenary status can be determined based on this.

Therefore, the establishment of the principal component analysis control chart is divided into two processes. First of all, the detection data are used to construct a principal component analysis control chart model that can correctly evaluate the status of the catenary, determine the number of principal components required, and calculate the control limits of the $T^{2}$ control chart and the SPE control chart. Then, on the basis of the control chart, the detection data of other sections of the catenary is analyzed and evaluated, and the corresponding principal component contribution control chart is drawn for the over-limit point to diagnose the cause.

\section{Case Analysis}

Take the detection data of a certain section of the catenary and use the multivariate statistical control chart to evaluate and diagnose the state of the catenary. Select the lead height $X 1$, the pull-out value $X 2$, the height difference $X 3$, the hard point $X 4$, and the contact force $X 5$ in the detection parameters as the detection parameters for judging the state of the catenary and constitute the detection parameter $X=(X 1$, $X 2, X 3, X 4, X 5)$. The changes of 25 groups of detection parameters of a certain section of catenary are shown in Figure 1. The red line in the figure is the allowable value range determined in accordance with the current "Highspeed Railway Catenary Operation and Maintenance Rules".

Table 1 shows the standardization value of the catenary detection parameters in this section.

Calculate the eigenvalues and eigenvectors of the standardized data and arrange them in the descending order of eigenvalues. Calculate the cumulative sum of different eigenvalues, take the cumulative sum criterion $C_{R}=85 \%$, and the cumulative contribution rate of the first three principal components is $87 \%$. Therefore, the number of principal components is determined to be three, and only the first three principal components can include most of the information in the analysis, so as to achieve the purpose of dimensionality reduction.

According to (9) and (11), the dot values of $T^{2}$ control chart and SPE control chart are calculated, as shown in Table 2.

Given the confidence level $\alpha=0.01$, it is calculated according to (10) that $T^{2} U C L=13.50$, calculate that $\theta_{1}=\sum_{j=4}^{5} \lambda_{j}=0.65, \theta_{2}=\sum_{j=4}^{5} \lambda_{j}^{2}=0.29, \theta_{3}=\sum_{j=4}^{5} \lambda_{j}^{3}=0.14$, $h_{0}=1-2 \theta_{1} \theta_{3} / 3 \theta_{2}^{2}=0.25$. According to (12), the SPE control limit is calculated as $Q U C L=0.61$. Plot the dot values and control limits of the principal component $T^{2}$ control chart, SPE control chart, and conventional multivariate $T^{2}$ control chart mentioned in this article in the same graph, and the multivariate statistical control chart of the catenary obtained is shown in Figure 2.

It can be seen from Figure 2 that the multivariate statistical control chart can integrate the multidimensional detection parameters of the catenary into a statistical quantity and visually display it in the form of graphics, which makes it easier to judge the state of the catenary. For this section of the catenary, the statistics of its state parameters are all within the control limit, indicating that the detection data $X$ are in a controlled state, and the state of this section of the catenary is normal. At the same confidence level, the principal component $T^{2}$ control chart in this paper retains the core principal components and is more sensitive to the fluctuation of the detection parameters than the multivariate $T^{2}$ control chart.

Select the detection data of this section of the catenary in another time period and draw the corresponding principal component analysis control chart, as shown in Figure 3.

It can be seen from Figure 3 that the statistics of the 13th group of the $T^{2}$ control chart are abnormal, and the statistics of the 13th and 25th groups of the SPE control chart are abnormal. The 13th set of data is abnormal in both the principal component subspace and the error subspace, indicating that the state reflected in this set of data is abnormal and needs to be adjusted. The abnormality of the 25 th group of statistics reflects that the data have a large deviation from the principal component space, the correlation between the data has undergone abnormal changes, and the contact network status is abnormal and needs to be adjusted.

In order to find out the reasons for the abnormality of statistics in the 13 th and 25 th groups, the contribution rate of the abnormal parameter is calculated as shown in Figure 4.

As can be seen in Figure 4, the hard point and height difference in the 13th group have a larger contribution rate, and the 25th group hard point and the leading height have a larger contribution rate. Based on this, it is judged that the state at the position of the catenary reflected by the 13th group of statistics is abnormal, and the factors that cause the abnormal state at this point are hard point and height difference. The factor causing the abnormality of the 25th group of statistics is the change in the correlation between the hard point and the height difference.

Combined with the actual detection parameter changes, the analysis in Figure 1 shows that the principal component analysis control chart does not need to analyse the detection parameters in each detection group separately and can comprehensively evaluate all the detection parameters to evaluate the catenary status. Not only it is more sensitive to data fluctuations, it can detect abnormalities in advance, and it can also detect abnormalities caused by parameter out-oflimits and changes in related relationships. The principal component analysis control chart can not only reflect the 


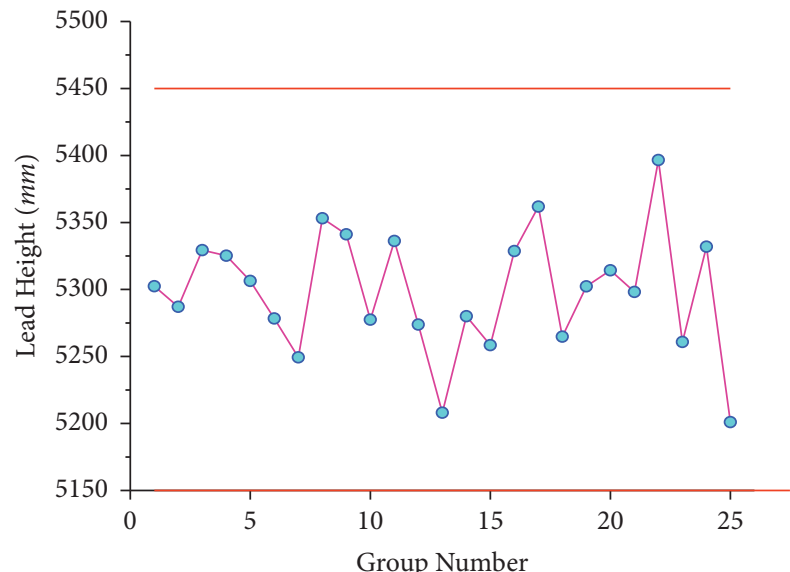

(a)

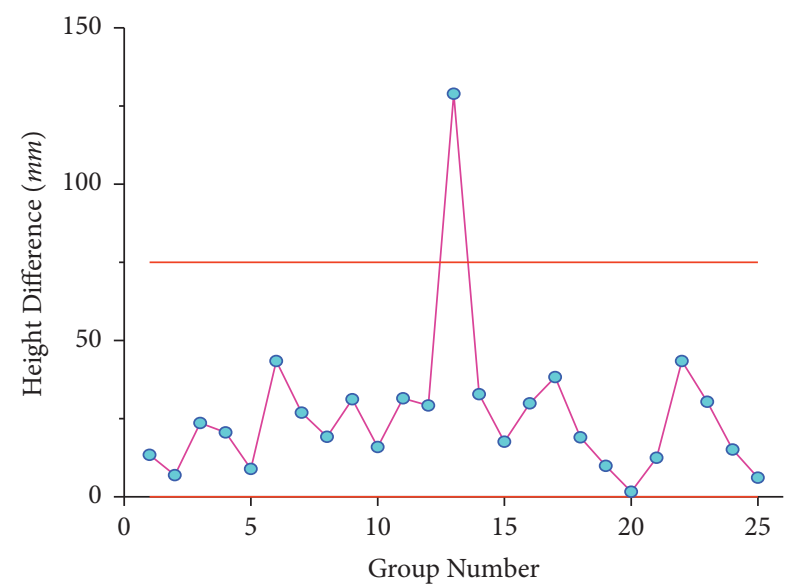

(c)

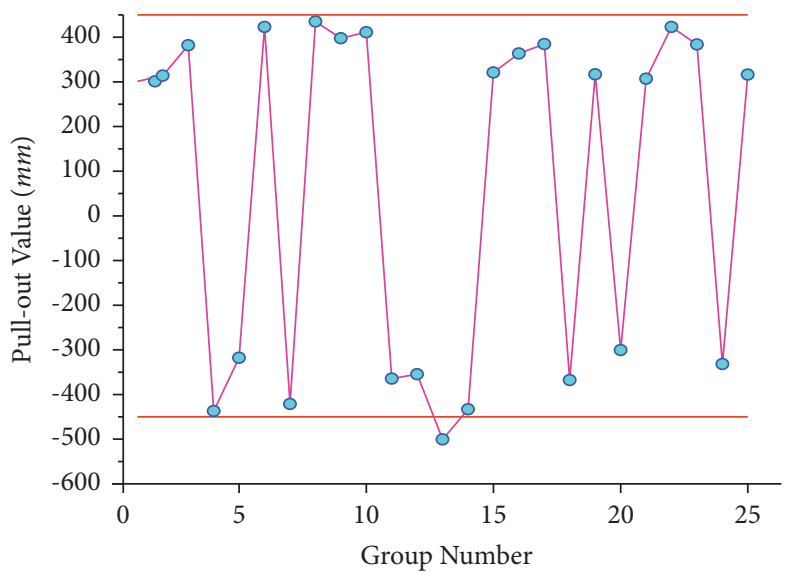

(b)

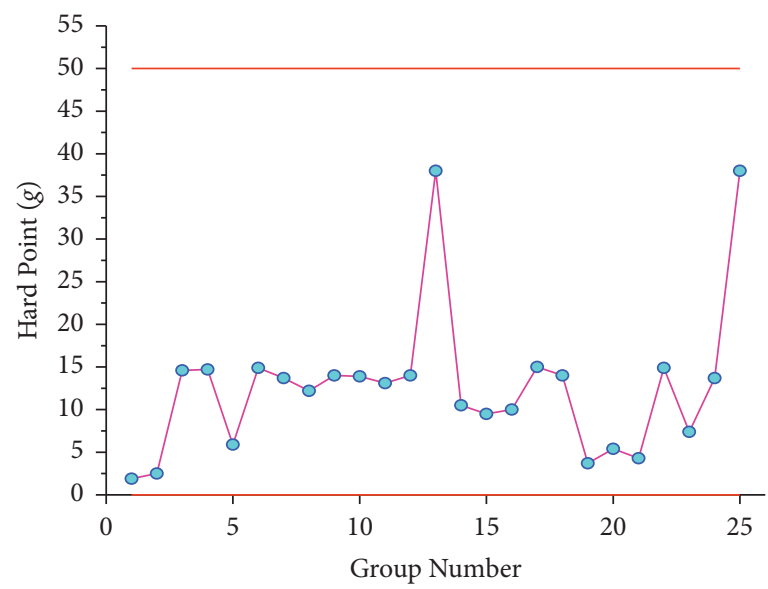

(d)

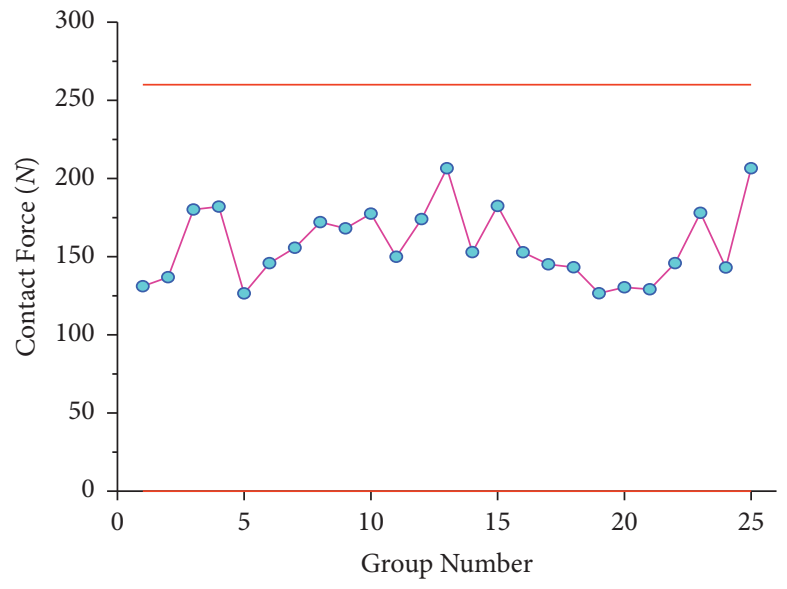

(e)

FIgURe 1: Changes of catenary parameters of one section. (a) Lead Height/mm; (b) pull-out value/mm; (c) height difference/mm; (d) hard point/(g); (e) contact force/(N). 
TABLE 1: Standardized data of the catenary detection parameters in this section.

\begin{tabular}{|c|c|c|c|c|}
\hline $\mathrm{X} 1$ & $\mathrm{X} 2$ & $\mathrm{X} 3$ & $\mathrm{X} 4$ & $\mathrm{X} 5$ \\
\hline 0.33 & 0.92 & -0.68 & -0.68 & -0.96 \\
\hline 0.40 & 0.84 & -0.95 & -1.00 & -1.05 \\
\hline 0.09 & -0.73 & -0.79 & -0.88 & -0.67 \\
\hline 1.19 & -0.98 & -0.72 & -0.49 & -0.71 \\
\hline-0.60 & 0.87 & 0.54 & -0.60 & -0.63 \\
\hline 0.42 & 0.92 & 1.93 & 2.11 & -0.36 \\
\hline-0.22 & -0.71 & -0.99 & 0.18 & 0.79 \\
\hline 1.64 & -1.15 & 0.55 & 2.39 & 1.38 \\
\hline-0.56 & 1.18 & 0.14 & -0.21 & -0.26 \\
\hline 0.44 & -0.76 & -1.32 & -0.83 & -0.70 \\
\hline 0.47 & -0.78 & -0.91 & -0.95 & -0.43 \\
\hline 0.27 & -0.74 & -0.53 & -0.86 & -1.04 \\
\hline-0.08 & 1.03 & 0.23 & 0.70 & 2.81 \\
\hline-0.27 & -1.11 & -0.43 & -0.24 & 0.75 \\
\hline 0.96 & 1.14 & 1.53 & 0.25 & -0.06 \\
\hline-1.00 & -1.16 & 0.23 & -0.23 & 0.73 \\
\hline-1.68 & -1.22 & 1.87 & 0.19 & -0.30 \\
\hline-2.05 & -0.93 & 0.11 & -0.06 & 0.29 \\
\hline 1.42 & 1.17 & -0.27 & 0.05 & 0.78 \\
\hline 0.87 & 1.00 & 1.11 & 0.36 & -0.29 \\
\hline-2.17 & -1.07 & 1.34 & 2.55 & 2.14 \\
\hline-1.09 & 1.28 & 1.01 & 0.08 & -0.07 \\
\hline 0.36 & 0.88 & -0.87 & -0.73 & -0.99 \\
\hline 0.49 & 0.82 & -0.92 & -0.74 & -0.36 \\
\hline 0.36 & -0.72 & -1.22 & -0.38 & -0.80 \\
\hline
\end{tabular}

TABLE 2: Rated value of $T^{2}$ control chart and SPE control chart.

\begin{tabular}{lccccc}
\hline Group no. & $T^{2}$ & $Q$ & Group no. & $T^{2}$ \\
\hline 1 & 3.40 & 0.14 & 14 & 1.61 & 3.15 \\
2 & 2.42 & 0.12 & 15 & 1.80 & 0.11 \\
3 & 4.13 & 0.18 & 16 & 6.36 \\
4 & 5.72 & 0.21 & 17 & 4.63 & 0.20 \\
5 & 2.11 & 0.01 & 18 & 0.43 \\
6 & 7.31 & 0.49 & 19 & 0.02 \\
7 & 1.86 & 0.13 & 20 & 0.00 \\
8 & 9.68 & 0.01 & 21 & 9.46 & 0.00 \\
9 & 2.00 & 0.31 & 22 & 0.16 \\
10 & 2.91 & 0.07 & 23 & 0.18 \\
11 & 1.59 & 0.07 & 24 & 1.32 \\
12 & 2.49 & 0.00 & 25 & 0.26 \\
13 & 8.48 & 0.04 & & 0.52 \\
\hline
\end{tabular}

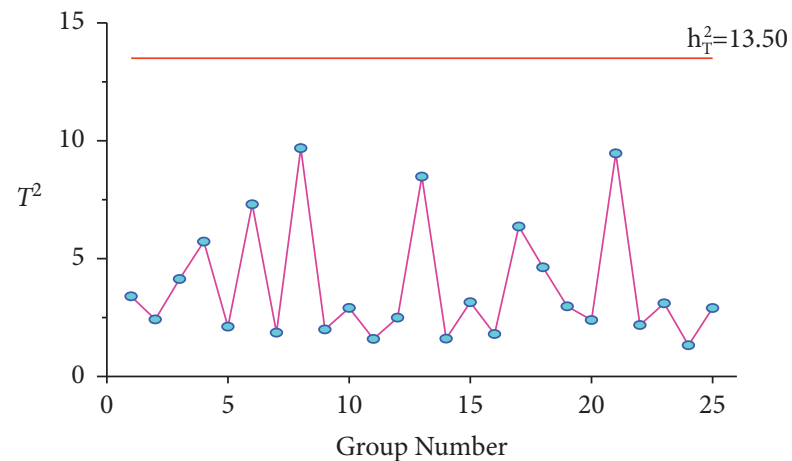

(a)

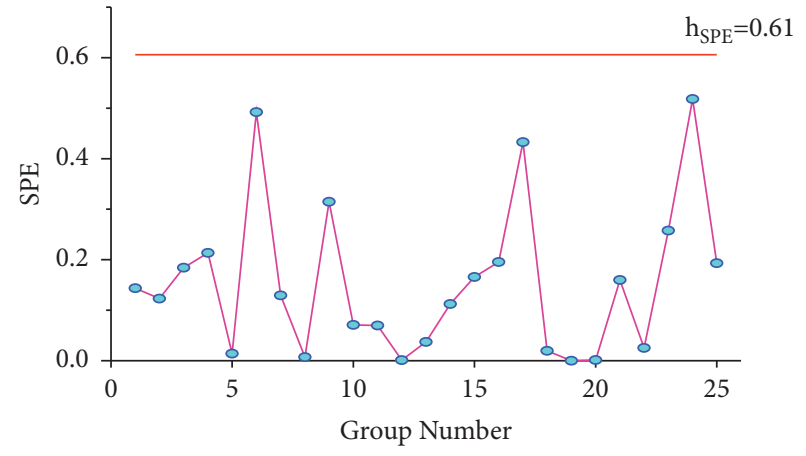

(b)

Figure 2: Continued. 


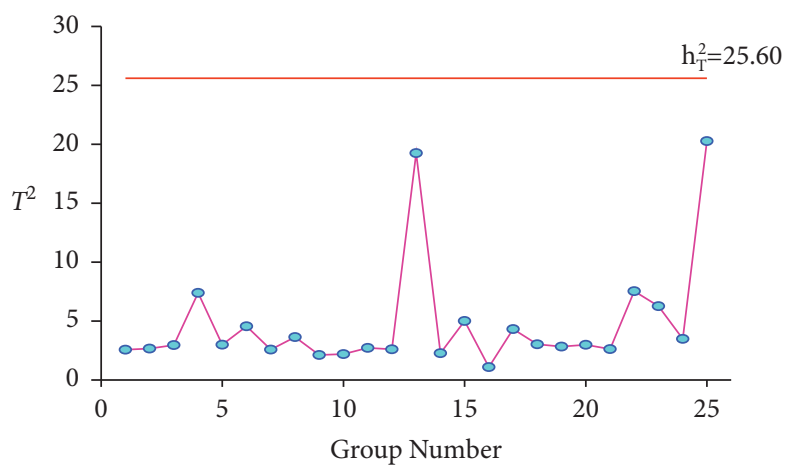

(c)

Figure 2: $T^{2}$ control chart and SPE control chart of catenary in normal states. (a) Principal component $T^{2}$ control chart; (b) SPE control chart; (c) conventional multiple $T^{2}$ control chart.

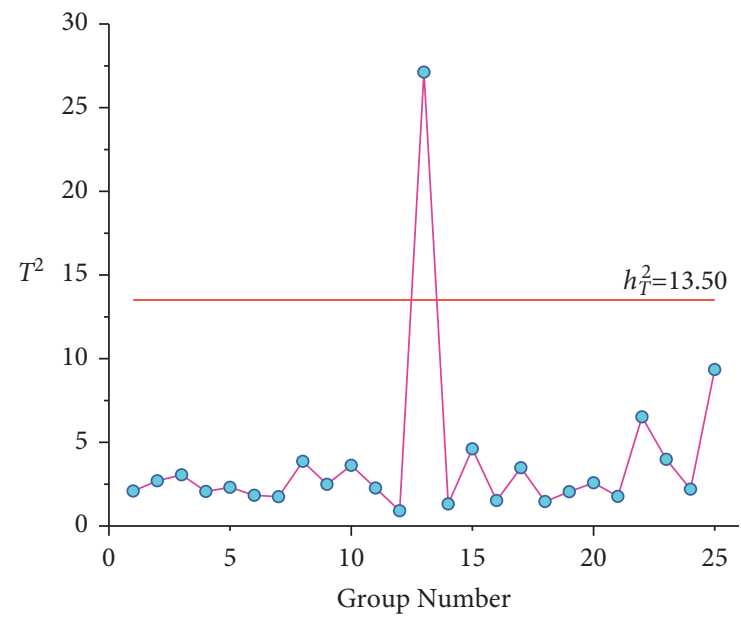

(a)

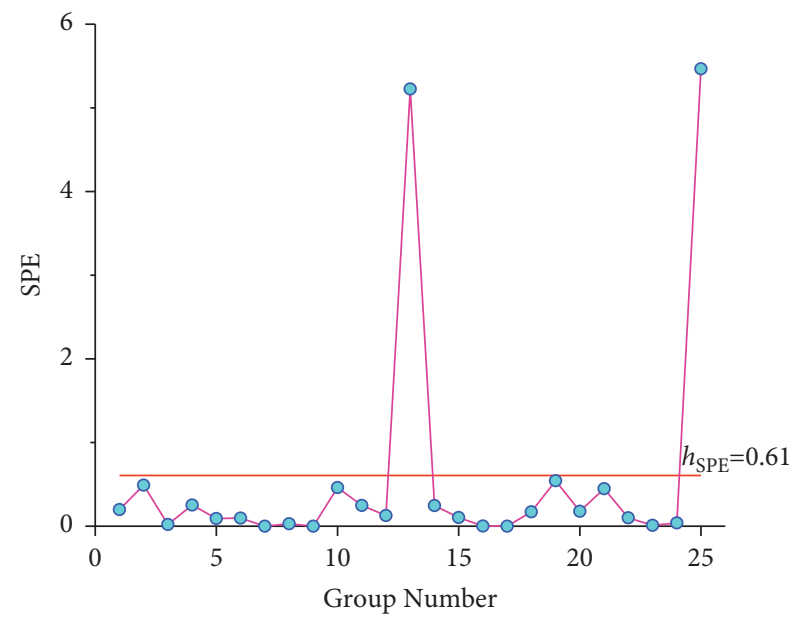

(b)

Figure 3: $T^{2}$ control chart and SPE control chart of catenary in abnormal states. (a) Principal component $T^{2}$ control chart; (b) SPE control chart.

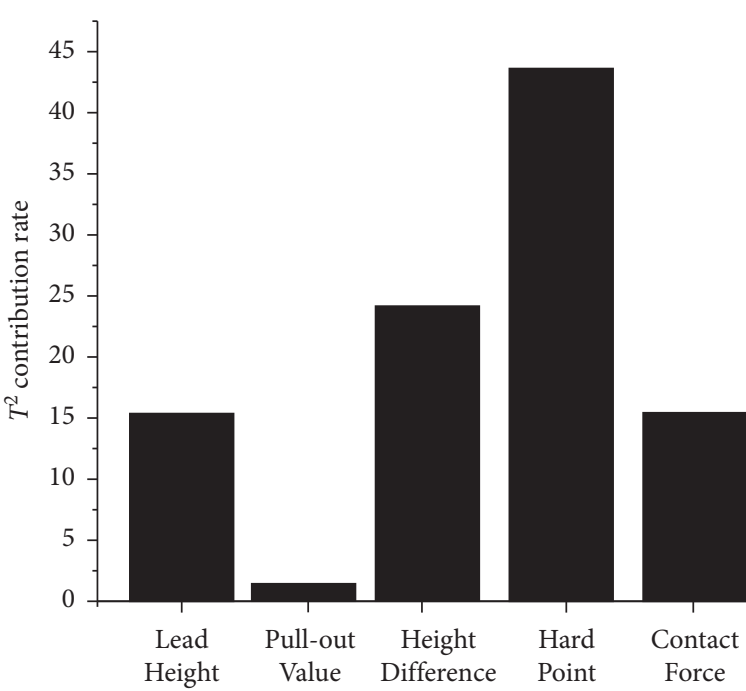

(a)

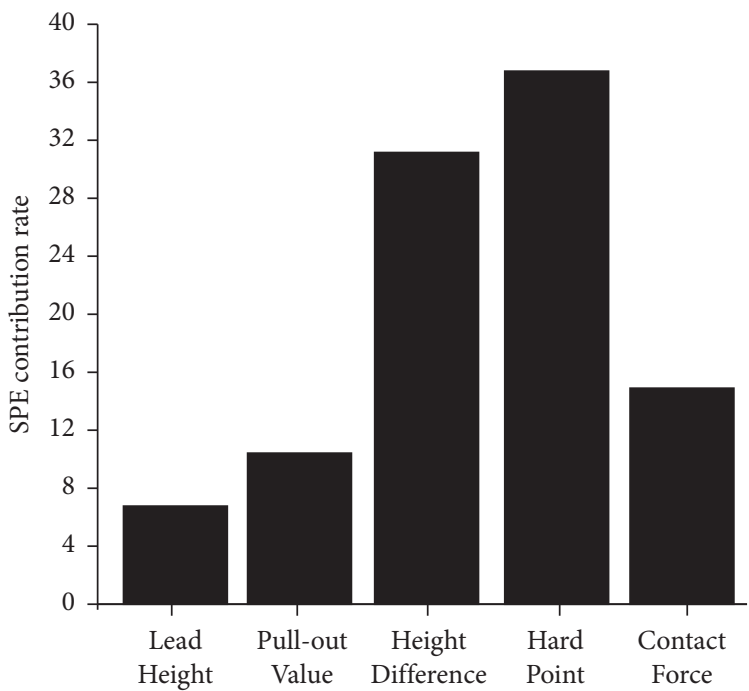

(b)

Figure 4: Continued. 


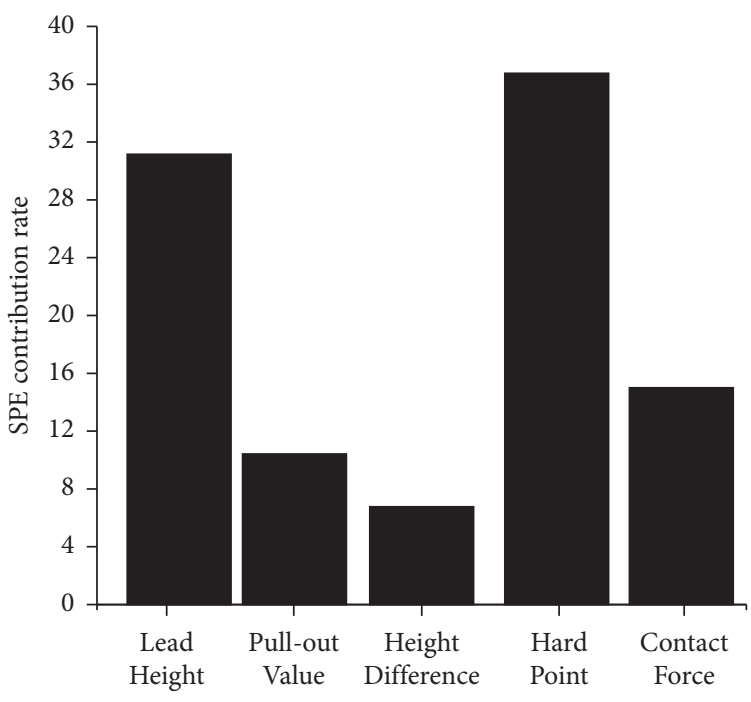

(c)

FIGURE 4: Contribution control chart of group 13 and group 25. (a) $T^{2}$ contribution rate chart of group 13; (b) SPE contribution rate chart of group 13; (c) SPE contribution rate chart of group 25.

changes in the principal component space, show the correlation within the data, but also find out the cause of the failure.

\section{Conclusion}

(1) The main component $T^{2}$ control chart can show the degree of fluctuation and deviation of the catenary status parameters. The residual SPE control chart can reflect the changes in the relationship of the catenary state parameters. The main component $T^{2}$ control chart and the residual SPE control chart of the catenary parameters can be used to evaluate the state of the catenary.

(2) The principal component $T^{2}$ control chart and the residual SPE control chart can convert the multidimensional detection parameters of the catenary into a statistic. It is displayed in the form of graphs, and the relationship between statistics and control limits is used to judge the status of the catenary. The method is simple and the result is intuitive.

(3) The contribution rate control chart can reflect the contribution degree of different parameters to the abnormal state on the basis of the main component $T^{2}$ control chart and the residual SPE control chart to realize the abnormal judgment of the catenary state. It can be used to determine the cause of the abnormal state of the catenary, so as to provide targeted guidance for the maintenance of the catenary.

\section{Data Availability}

The data sets used or analyzed during the current study are available from the corresponding author on reasonable request.

\section{Conflicts of Interest}

The authors declare that there are no conflicts of interest regarding the publication of this paper.

\section{Acknowledgments}

This work was supported by the research project of Guangzhou Metro Design and Research Institute Co., Ltd., under grant KY-2021-006.

\section{References}

[1] F. Lin, J. Luo, and Z. Li, "Statistical analysis of national electric traction power supply system failures in 2003 and 2004," Gansu Science and Technology, vol. 2006, no. 3, pp. 27-28, 2006.

[2] J. Zhou and C. Wu, "Analysis of high-speed railway catenary detection technology," China High-Tech Enterprise, vol. 23, no. 3, pp. 110-111, 2017.

[3] W. Zhou, Z. Sun, S. Ren et al., "Measuring method of geometric parameters of catenary based on multi-eye stereo vision," China Railway Science, vol. 36, no. 5, pp. 104-109, 2015.

[4] Z. Li, Research on Non-contact Detection Technology of Catenary Parameters, East China Jiaotong University, Nanchang, China, 2017.

[5] D. Zhu, "German catenary dynamic detection technology," Electric Railways, vol. 17, no. 3, pp. 13-14, 2004.

[6] X. Zhao, X. Wu, and G. Xu, "German high-speed railway catenary detection system," China Railway, vol. 38, no. 9, pp. 60-62, 2008.

[7] H. Bai, Research on the Detection of Catenary Suspension Status of High-Speed Railway, China Academy of Railway Sciences, Beijing, China, 2016.

[8] R. Zhang, Z. Liu, and Z. Yang, "Research on 6C system information comprehensive application technology scheme," China Railway, vol. 48, no. 6, pp. 44-50, 2018. 
[9] R. Zhang and Z. Yang, "Research and practice of monitoring system for catenary operation state," China Railway, vol. 49, no. 9, pp. 64-70, 2019.

[10] Ministry of Railways of the People's Republic of China, General Technical Specifications for the High-Speed Railway Power Supply Safety Detection and Monitoring System (6C System)Power Supply Department, Beijing, China, 2012.

[11] China National Railway Group Co., Ltd, "The outline of the advanced railway planning for a powerful country in the new era," People's Railway, no. 2, 2020.

[12] National Development and Reform Commission, Mid- to Long-Term Railway Network Planning, National Development and Reform Commission, Beijing, China, 2016.

[13] China Railway Corporation, High-speed Railway Catenary Technology, China Railway Publishing House, Beijing, China, 2014.

[14] Q. Qian, S. Gao, Z. He, Q. Chen, and J. Wu, "Key technologies of traction power supply for high-speed railways in China," China Engineering Science, vol. 17, no. 4, pp. 9-20, 2015.

[15] J. Wang, "Research on determining the weights of influencing factors of catenary section quality evaluation based on analytic hierarchy process," China Railway, vol. 49, no. 4, pp. 60-64, 2019.

[16] H. Cheng, Z. He, H. Hu, B. Wang, and X. Sun, "Entropy weight multi-information comprehensive assessment of the health status of high-speed rail catenary," Journal of the China Railway Society, vol. 36, no. 3, pp. 19-24, 2014.

[17] Yi Yin, S. Gao, and M. Wang, "Feature extraction of catenary parameters of high-speed railway," Electric Railways, vol. 29, no. 5, pp. 29-32, 2018.

[18] S. Liu, X. Zhu, Y. Zhang, and L. Wu, "Research on the health status assessment of high-speed railway catenary based on grey clustering and combined weighting method," Journal of the China Railway Society, vol. 38, no. 7, pp. 57-63, 2016.

[19] D. Zhang, High-speed Railway Catenary Status Assessment Based on Detection Parameters, Southwest Jiaotong University, Chengdu, China, 2019.

[20] S. Liu, Z. Chou, and Z. Ma, "Comprehensive evaluation of catenary health status based on variable weight matter element extension model," Journal of East China Jiaotong University, vol. 36, no. 1, pp. 125-132, 2019.

[21] Ye Tian, Application of Data Mining Technology in Data Processing of Catenary Detection, Southwest Jiaotong University, Chengdu, China, 2005.

[22] H. Wang and K. Feng, "High-speed railway catenary system safety evaluation based on structural entropy weight method and cloud model," China Railway, vol. 49, no. 12, pp. 41-46, 2019.

[23] M. Zhang, H. Cheng, X. Wang, and Y. Li, "Evaluation of catenary operation status based on improved variable weight method and cloud model," Journal of Railway Science and Engineering, vol. 13, no. 5, pp. 964-970, 2016.

[24] D. Zhang, Z. Han, Z. Liu, S. Gao, and H. Wei, "Assessment of the health status of the high-speed rail catenary based on set pair analysis and evidence theory," Journal of the China Railway Society, vol. 42, no. 5, pp. 58-65, 2020.

[25] X. Xu and L. Ma, "Control chart recognition based on transfer learning and convolutional neural network," Computer Applications, vol. 38, no. S2, pp. 290-295, 2018.

[26] J. Zhao, "The application of statistics in quality control," Statistics and Management, vol. 35, no. 5, pp. 6-7, 2015.

[27] J. Jiang, X. Jiang, Y. Feng, and S. Deng, "Comparative study of multivariate statistical control charts," Aviation Standardization and Quality, no. 1, pp. 17-21, 2017.
[28] Y. Qin, H. Huang, F. Jian, and Y. Chen, "Transformer fault diagnosis based on FCM and improved PC," High Voltage Apparatus, vol. 54, no. 12, pp. 262-267, 2018.

[29] B. Wen, Research on Fault Detection and Diagnosis Based on Principal Component Analysis, Nanjing Normal University, Nanjing, China, 2011.

[30] C. Wen, F. Lv, Z. Bao, and M. Liu, "Overview of data-driven micro-fault diagnosis methods," Acta Automatica Sinica, vol. 42, no. 9, pp. 1285-1299, 2016.

[31] Y. Chang, S. Wang, F. Wang, and J. Fu, "Process monitoring method based on multi-PCA model," Chinese Journal of Scientific Instrument, vol. 35, no. 4, pp. 901-908, 2014.

[32] Z. Xiao and X. Ran, "Process control and diagnosis using principal component analysis," Journal of Chongqing University of Technology (Natural Science), vol. 28, no. 1, pp. 96-101, 2014. 